\title{
Hopf Bifurcation Analysis for a Stochastic Discrete-Time Hyperchaotic System
}

\author{
Jie Ran, ${ }^{1}$ Yu-Qin Li, ${ }^{2}$ Shao-Juan Ma, ${ }^{3}$ and Juan $\mathrm{Wu}^{3}$ \\ ${ }^{1}$ Institute of Mathematics and Computer Science, Zunyi Normal University, Zunyi 563000, China \\ ${ }^{2}$ Department of Basic Education, Business School of Guizhou University of Finance and Economics, Huishui 550600, China \\ ${ }^{3}$ School of Mathematics and Information Science, North University of Nationalities, Yinchuan 750021, China \\ Correspondence should be addressed to Jie Ran; ranjie93@163.com
}

Received 6 October 2015; Revised 9 November 2015; Accepted 19 November 2015

Academic Editor: Daniele Fournier-Prunaret

Copyright (C) 2015 Jie Ran et al. This is an open access article distributed under the Creative Commons Attribution License, which permits unrestricted use, distribution, and reproduction in any medium, provided the original work is properly cited.

\begin{abstract}
The dynamics of a discrete-time hyperchaotic system and the amplitude control of Hopf bifurcation for a stochastic discrete-time hyperchaotic system are investigated in this paper. Numerical simulations are presented to exhibit the complex dynamical behaviors in the discrete-time hyperchaotic system. Furthermore, the stochastic discrete-time hyperchaotic system with random parameters is transformed into its equivalent deterministic system with the orthogonal polynomial theory of discrete random function. In addition, the dynamical features of the discrete-time hyperchaotic system with random disturbances are obtained through its equivalent deterministic system. By using the Hopf bifurcation conditions of the deterministic discrete-time system, the specific conditions for the existence of Hopf bifurcation in the equivalent deterministic system are derived. And the amplitude control with random intensity is discussed in detail. Finally, the feasibility of the control method is demonstrated by numerical simulations.
\end{abstract}

\section{Introduction}

The dynamics and their bifurcation control in chaos systems have been given much attention and widely used in chemical and biological population and power systems $[1,2]$. Studies about bifurcation mainly include the validated existence of bifurcation and its control. Hopf bifurcation has been researched comprehensively and systematically in theory [36]. The aim of bifurcation control is to design a controller to modify the bifurcation properties of a given nonlinear system and then achieve the other desirable dynamical behaviors. There are some summaries about bifurcation control, such as delayed-feedback control [7], state-feedback control [8], and Washout-filter control [9]. The condition of existing bifurcation and Hopf bifurcation for discrete-time systems is studied by Wen et al. $[10,11]$. The study of chaos has become a hot research topic recently. Compared to the chaotic system, hyperchaotic system has two positive Lyapunov exponents. Therefore, hyperchaotic system would have more complex dynamical behaviors than a normal chaotic system. In 1979, hyperchaotic system was firstly reported by Rössler system [12]. From then on, hyperchaotic system has received great attention in the past several decades [13-15]. The study of hyperchaotic system proposed for secure communication is much more significance than the chaos system. It is well known that there are many methods to control the chaotic and hyperchaotic systems, such as nonlinear feedback, statefeedback control [16], linear feedback and adaptive control [17], and time-delay feedback control [18]. There are advantages and disadvantages in each control method. The chaos and hyperchaos control based on the first control is studied by Ma and Yang in [19]. In practice, most researchers focus mainly on how to construct a hyperchaotic system and how to design hyperchaotic circuit [20]. As far as we know, there are few studies on hyperchaotic systems about their dynamical behaviors, such as bifurcation and chaos.

However, owing to the uncertain factors from external environment, uncertainties of system parameters, perturbations of external noise, and so on, the stochastic system can accurately represent the original systems better; 
therefore the study of stochastic system is more meaningful than deterministic systems. Nowadays, a growing number of researchers have shown great interests in the research of stochastic dynamics for nonlinear systems with random parameters. Orthogonal polynomial approximation based on the expansion theory of orthogonal polynomials has been widely used. The dynamical behaviors via the improved method in stochastic systems with random parameters are studied by Leng et al. [21]. Ma et al. have investigated the stochastic Hopf bifurcation [22-24]. Hopf bifurcation control for stochastic dynamical system with nonlinear random feedback method has been investigated [25]. From the previous research about stochastic model, those stochastic systems are always inevitably disturbed by the system parameters. Hence, the dynamics analysis of stochastic discrete-time hyperchaotic system with random parameter, which is scarcely investigated, attracts our interest.

The remainder of this paper is arranged as follows. In Section 2, the dynamics of a discrete-time hyperchaotic system are investigated, and a stochastic discrete-time hyperchaotic system transforms into its equivalent deterministic system by means of orthogonal polynomial approximation. Then, existence of Hopf bifurcation and bifurcation amplitude control with random intensity method are studied. And the numerical simulations are shown in Section 3 and in Section 4. Finally, conclusions are drawn in Section 5.

\section{Orthogonal Polynomial Approximation of a Discrete-Time Hyperchaotic System with Random Parameter}

Consider a two-dimensional deterministic discrete-time hyperchaotic system that has been researched by Chen:

$$
\begin{aligned}
& x(n+1)=a y(n)+b x(n)^{2}+c y(n)^{2}, \\
& y(n+1)=d x(n),
\end{aligned}
$$

where $x(n)$ and $y(n)$ are state variables and system parameters $a, b, c$, and $d$ are real parameters. Let $(b, c, d)=$ $(-0.1,-0.9,-0.48)$, varying $a$ in range $2<a<3.5$, system (1) has the corresponding bifurcation diagram, and Lyapunov exponents are depicted in Figure 1.

Numerical simulations are presented to exhibit the complex dynamical behaviors, such as period orbits, Hopf bifurcation, chaos, and hyperchaos. The phase portraits of system (1) are shown in Figure 2. These results reveal far richer dynamics of the discrete model compared with the chaos system. The numerically computed Lyapunov exponents and bifurcation diagram of system (1) can further confirm the complex dynamical behaviors.

It is well known that there exist many uncertain factors from external environment, manufacture, material, and installation; many dynamic systems are always inevitably affected by some random disturbances, such as uncertainties of system parameters, perturbations of external noise, and stochastic input. Hence, the stochastic system accurately represents the original system, and the dynamic behaviors analysis of stochastic system will possess more practical significance. The study of this kind of model is more significance than the deterministic system. In the following, we consider a stochastic discrete-time hyperchaotic system. Let $a, b$, and $c$ be deterministic parameters, and $d$ is a random parameter. Then the stochastic discrete-time hyperchaotic system can be written as the following system:

$$
\begin{aligned}
& x(n+1)=a y(n)+b x(n)^{2}+c y(n)^{2}, \\
& y(n+1)=(\bar{d}+\delta k) x(n)
\end{aligned}
$$

where $\bar{d}$ is the statistic parameter of $d$ in system (2), $\delta$ is regarded as strength of random disturbance, and $k$ is a random variable defined on nonnegative set integer which obeys density function $p_{k}$.

According to orthogonal polynomial approximation of discrete random function in the Hilbert spaces and the orthogonality of orthogonal polynomials [21-25], the response of discrete-time hyperchaotic system with random parameter can be expressed by the following Fourier series:

$$
\begin{aligned}
& x(n, k)=\sum_{i=0}^{M} x_{i}(n) P_{i}(k), \\
& y(n, k)=\sum_{i=0}^{M} y_{i}(n) P_{i}(k),
\end{aligned}
$$

where $x_{i}(n)=\sum_{i=0}^{N} p_{k} x(n, k) P_{i}(k), y_{i}(n)=\sum_{i=0}^{N} p_{k} y(n$, k) $P_{i}(k), P_{i}(k)$ is the orthogonal polynomial, and $M$ represents the largest order of the polynomial we have taken. Substituting (3) into (2), we have

$$
\begin{aligned}
\sum_{i=0}^{M} x_{i}(n+1) P_{i}(k)= & a\left(\sum_{i=0}^{M} y_{i}(n) P_{i}(k)\right) \\
& +b\left(\sum_{i=0}^{M} x_{i}(n) P_{i}(k)\right)^{2} \\
& +c\left(\sum_{i=0}^{M} x_{i}(n) P_{i}(k)\right)^{2}, \\
\sum_{i=0}^{M} y_{i}(n+1) P_{i}(k)= & (\bar{d}+\delta k)\left(\sum_{i=0}^{M} x_{i}(n) P_{i}(k)\right) .
\end{aligned}
$$

The cycle recurrence formula of polynomials is as follows:

$$
\begin{aligned}
k P_{i}(k)=\alpha_{i} P_{i+1}(k)+\beta_{i} P_{i}(k)+\gamma_{i} P_{i-1}(k), & \\
& \gamma_{i} \neq 0, P_{-1}(k)=0, P_{0}(k)=1,
\end{aligned}
$$

where $\alpha_{i}, \beta_{i}$, and $\gamma_{i}$ are decided by different kinds of polynomials.

In this paper, we assume that the random variable $k$ obeys the Poisson distribution with a parameter of $\lambda$. Corresponding to this random variable, the orthogonal polynomial is chosen as the Charlier polynomial. Therefore, the coefficients of (5) are $\alpha_{i}=1, \beta_{i}=i+\lambda$, and $\gamma_{i}=\lambda i$. When $M \rightarrow \infty$, the discrete-time hyperchaotic system with random parameter 


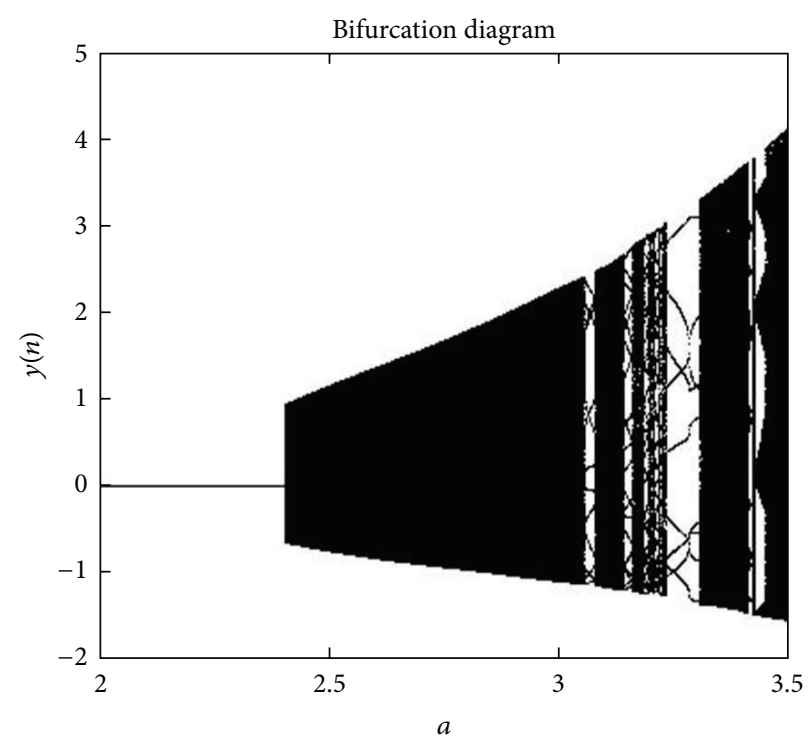

(a)

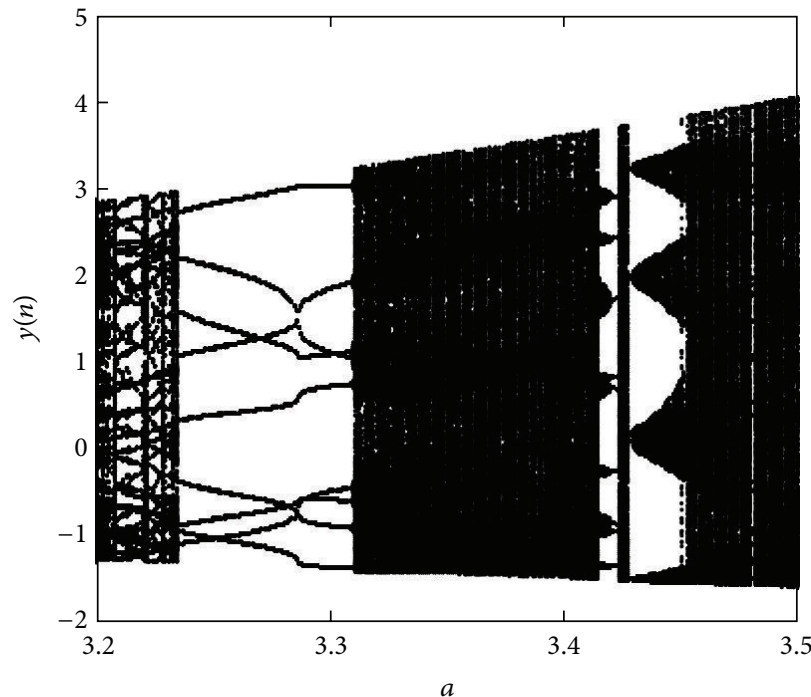

(b)

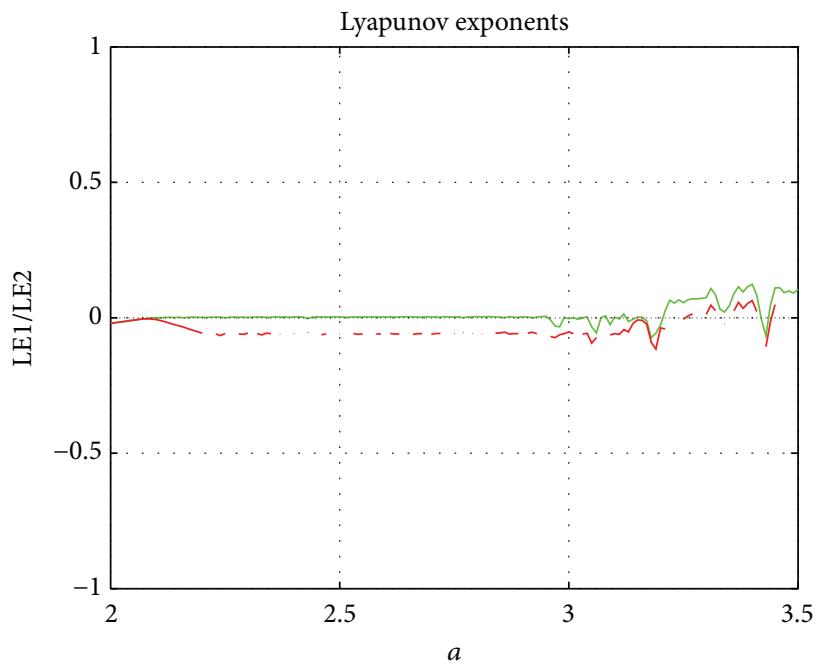

(c)

FIGURE 1: (a) Bifurcation diagram of system (1) when initial values are $x(1)=0.01$ and $y(1)=0.01$. (b) Local amplification corresponding to (a). (c) Lyapunov exponents corresponding to (a).

is strictly equivalent to system (4). In order to facilitate the numerical analysis of this paper, we select $M=1$ and $\gamma=1$ and approximately obtain the equivalent deterministic system of discrete-time hyperchaotic system with random parameter:

$$
\begin{aligned}
& x_{0}(n+1)=a y_{0}(n)+S_{0}(n)+U_{0}(n), \\
& y_{0}(n+1)=\bar{d} x_{0}(n)+\delta x_{0}(n), \\
& x_{1}(n+1)=a y_{1}(n)+S_{1}(n)+U_{1}(n), \\
& y_{1}(n+1)=\bar{d} x_{1}(n)+\delta\left(2 x_{1}(n)+x_{0}(n)\right),
\end{aligned}
$$

where $S_{i}(n)(i=0,1)$ and $U_{i}(n)(i=0,1)$ can be derived through the MAPLE (see Appendix). We can obtain the numerical solutions $x_{i}(n)$ and $y_{i}(n)$ of equivalent deterministic system (6) by effective numerical methods. Furthermore, the approximate random response of the original stochastic discrete-time hyperchaotic system can be expressed as

$$
\begin{aligned}
& x(n, k)=x_{0}(n) P_{0}(k)+x_{1}(n) P_{1}(k), \\
& y(n, k)=y_{0}(n) P_{0}(k)+y_{1}(n) P_{1}(k),
\end{aligned}
$$

and the ensemble mean response of system (2) is calculated as

$$
\begin{aligned}
E[x(n, k)] & =x_{0}(n) E\left[P_{0}(k)\right]+x_{1}(n) E\left[P_{1}(k)\right] \\
& =x_{0}(n),
\end{aligned}
$$




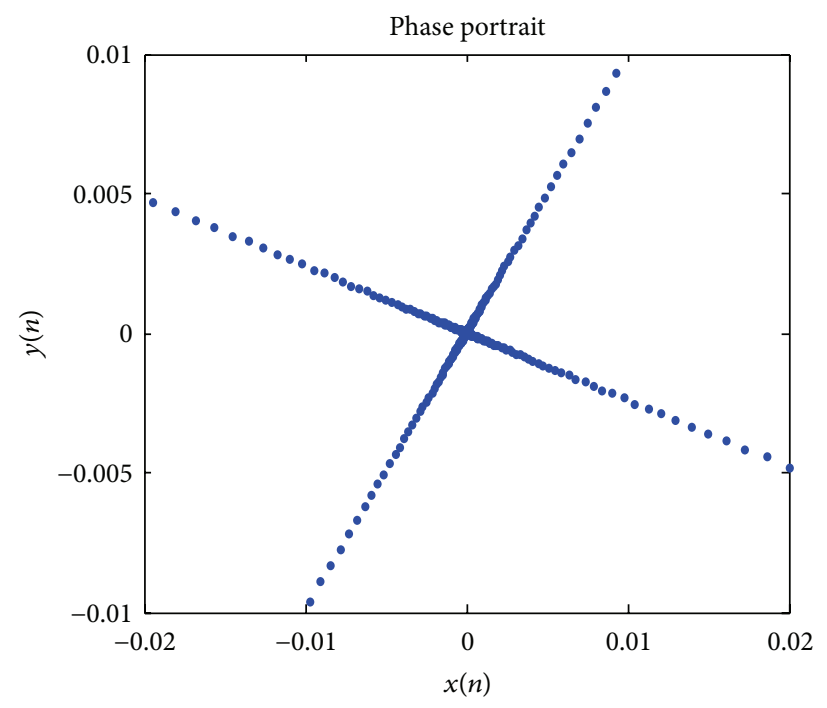

(a) $a=2.01$

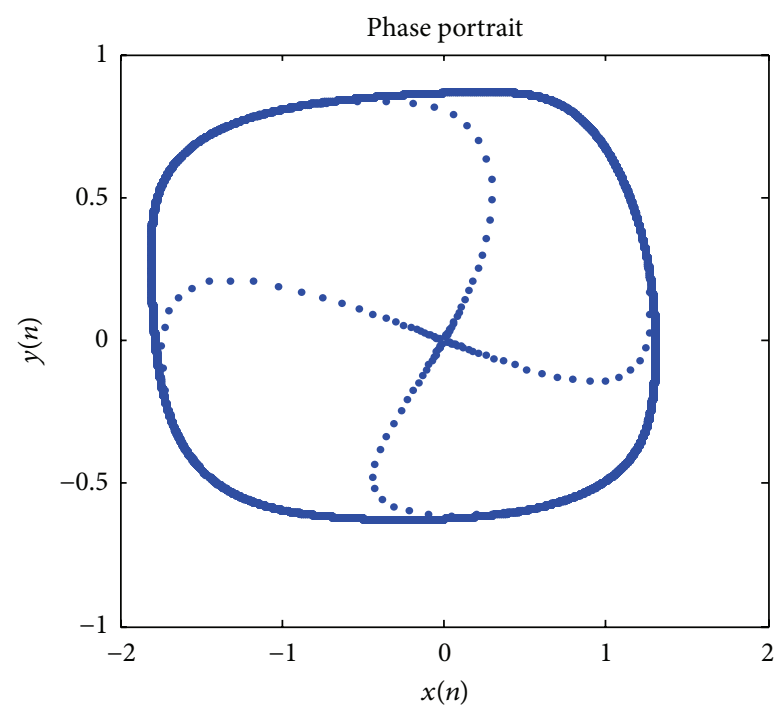

(c) $a=2.29$

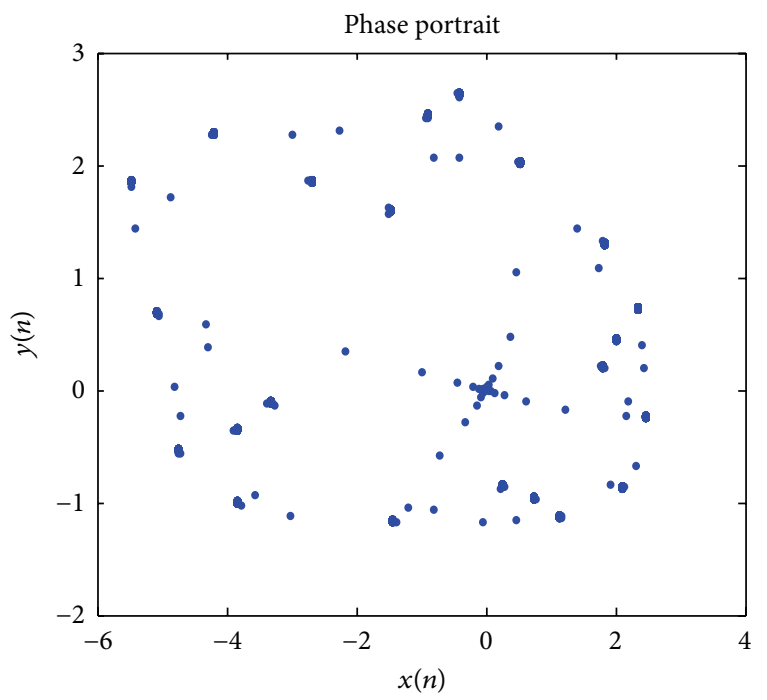

(e) $a=3.05$

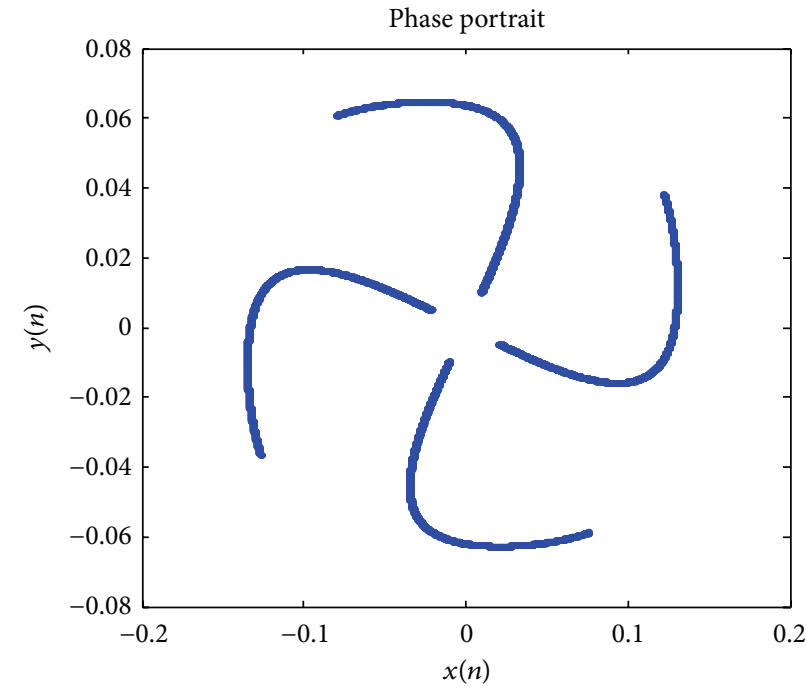

(b) $a=2.085$

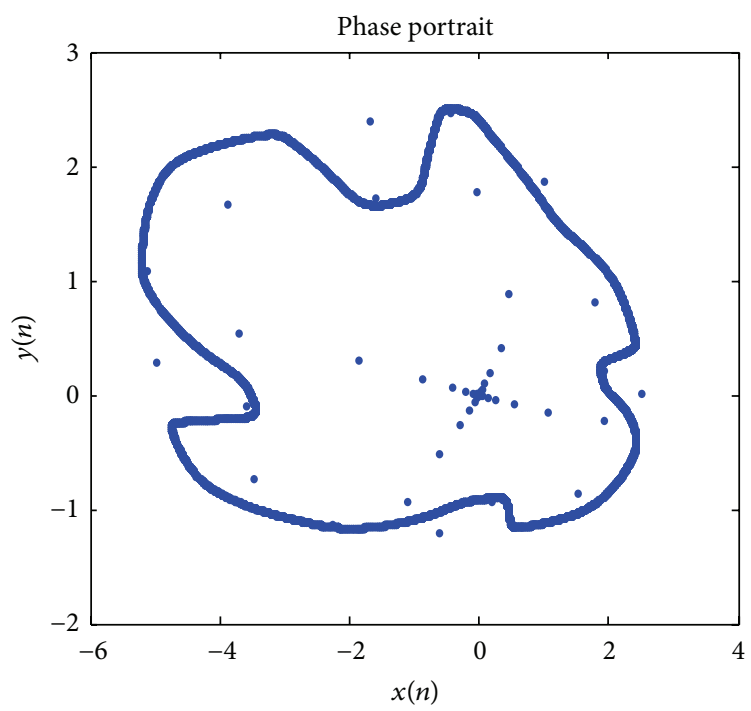

(d) $a=3.01$

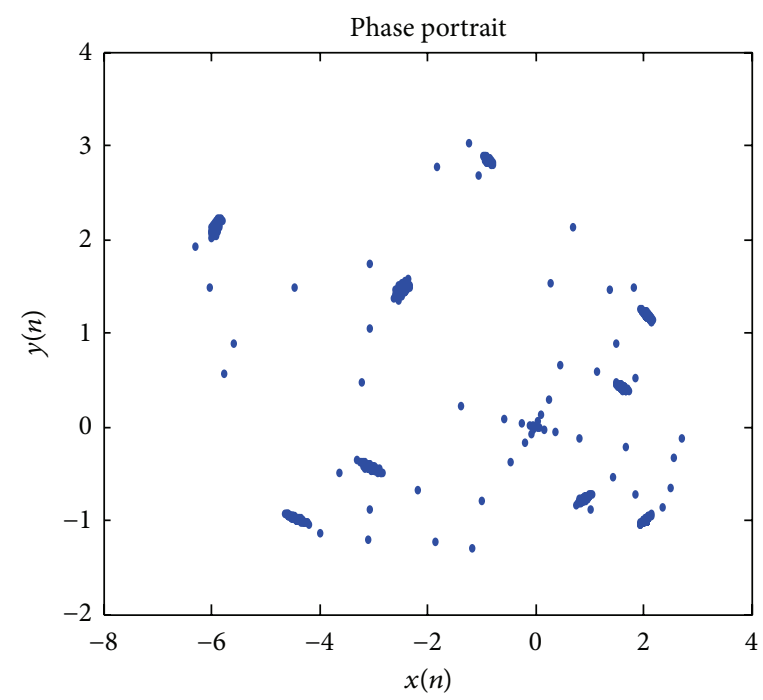

(f) $a=3.15$

Figure 2: Continued. 


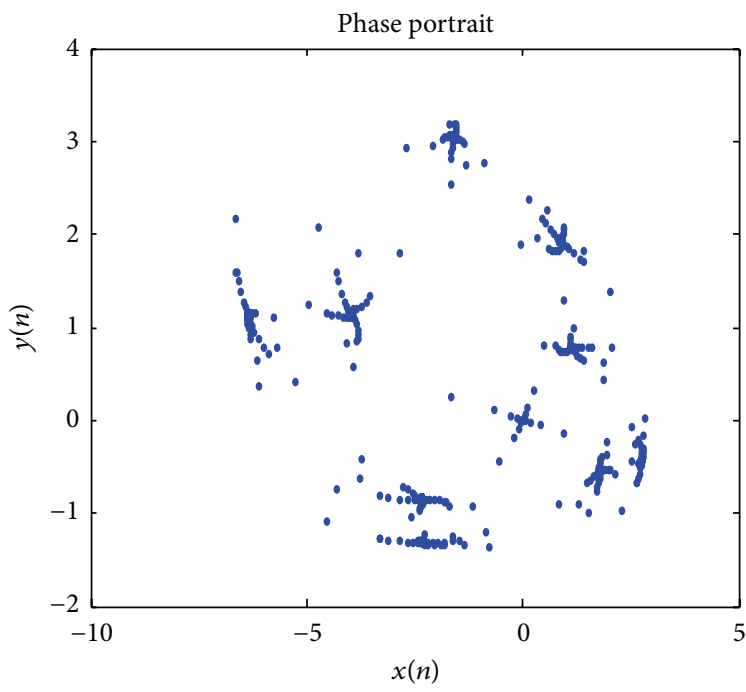

(g) $a=3.205$

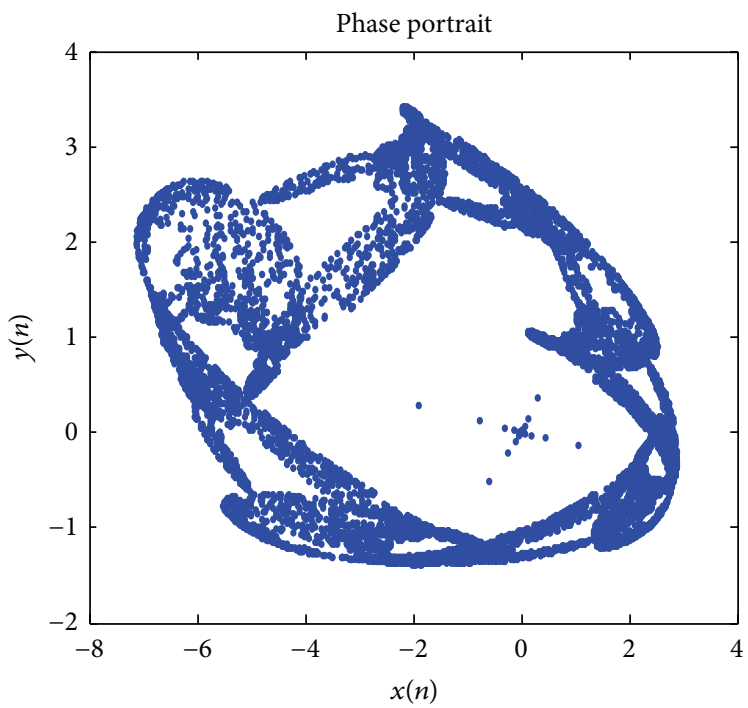

(i) $a=3.25$

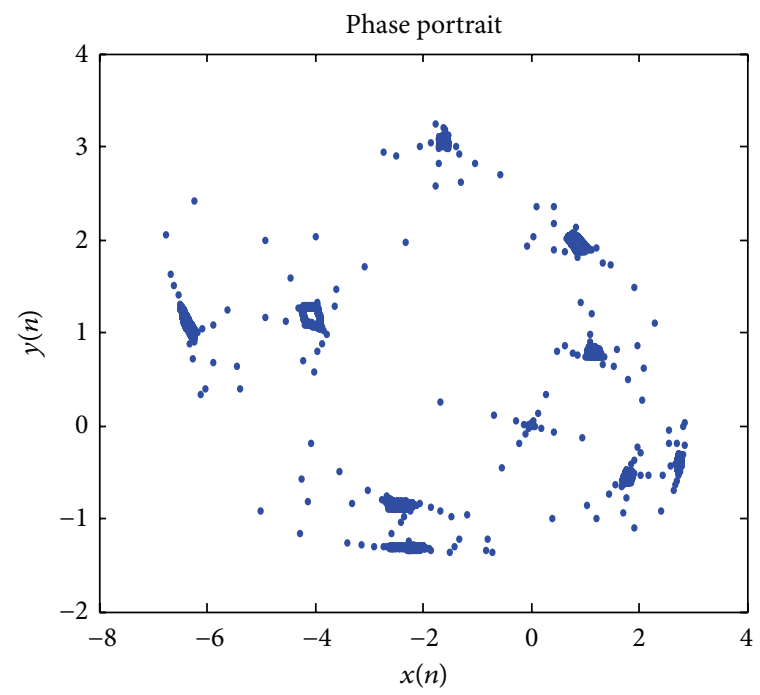

(h) $a=3.2101$

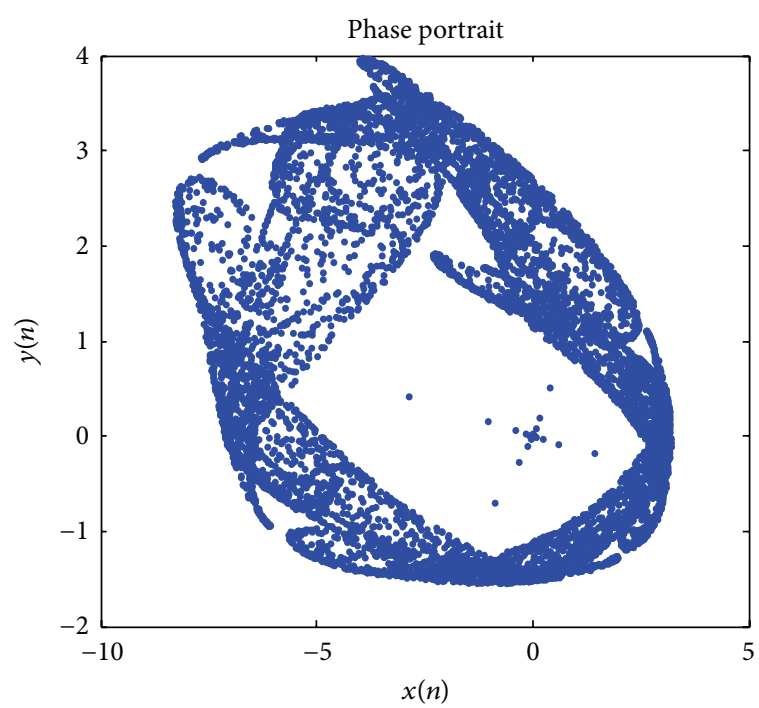

(j) $a=3.38$

FIGURE 2: Phase portraits of system (1) when parameter $a$ changes in the range as shown in Figure 1(a). The initial values of system (1) are $x(1)=0.01$ and $y(1)=0.01$.

$$
\begin{aligned}
E[y(n, k)] & =y_{0}(n) E\left[P_{0}(k)\right]+y_{1}(n) E\left[P_{1}(k)\right] \\
& =y_{0}(n) .
\end{aligned}
$$

The initial conditions of system (1) with deterministic parameters are defined as $x_{0}=x(0)$ and $y_{0}=y(0)$. In this paper we take the initial conditions of equivalent deterministic system (6) as follows:

$$
\begin{aligned}
& x_{0}=x(0)=0.01, \\
& y_{0}=y(0)=0.01, \\
& x_{1}=x(1)=0,
\end{aligned}
$$

$$
y_{1}=y(1)=0
$$

\section{Hopf Bifurcation Analysis}

In this section, we will investigate the Hopf bifurcation of discrete-time hyperchaotic system with random parameter.

Theorem 1. Stochastic discrete-time hyperchaotic system (2) undergoes the Hopf bifurcation in the strong resonance case at a fixed point, when the parameter $\bar{d}$ passes the critical value $\bar{d}_{c}=-1 / a-\delta$. 
Proof. The Jacobian matrix of equivalent deterministic system (6) at the zero fixed point is

$$
J=\left(\begin{array}{cccc}
0 & a & 0 & 0 \\
\bar{d}+\delta & 0 & 0 & 0 \\
0 & 0 & 0 & a \\
\delta & 0 & \bar{d}+2 \delta & 0
\end{array}\right)
$$

with the character polynomial

$$
f(\lambda)=b_{0} \lambda^{4}+b_{1} \lambda^{3}+b_{2} \lambda^{2}+b_{3} \lambda+b_{4}
$$

where $b_{i}(i=1, \ldots, 4)$ are coefficients of equivalent deterministic system (6), which are shown as follows:

$$
\begin{aligned}
& b_{0}=1, \\
& b_{1}=0, \\
& b_{2}=-3 a \delta-2 a \bar{d}, \\
& b_{3}=0, \\
& b_{4}=3 a^{2} \bar{d} \delta+2 a^{2} \delta^{2}+a^{2} \bar{d}^{2} .
\end{aligned}
$$

By a simple computation, it is straightforward to obtain the following results:

$$
\begin{aligned}
& \lambda_{1,2}= \pm \sqrt{a \bar{d}+a \delta} \\
& \lambda_{3,4}= \pm \sqrt{a \bar{d}+2 a \delta}
\end{aligned}
$$

The classical Hopf bifurcation criterion (see [11]) about the discrete-time system is repeated as follows: (C1) Eigenvalue assignment: the Jacobian matrix of the discrete-time system has a pair of complex conjugate eigenvalues, $\lambda_{1}(\mu)$ and $\bar{\lambda}_{1}(\mu)$ with $\left|\lambda_{1}(\mu)\right|=1$ at $\mu=\mu_{c}$ and the other eigenvalues $\lambda_{j}(\mu)$ $(j=3,4, \ldots, n)$, with $\left|\lambda_{j}\left(\mu_{c}\right)\right|<1$; (C2) transversality condition: $d\left|\lambda_{i}\left(\mu_{c}\right)\right| / d \mu \neq 0(i=1,2, \ldots, n)$; (C3) nonresonance condition: $\lambda_{1}^{m}(\mu) \neq 1$; resonance condition: $\lambda_{1}^{m}(\mu)=1$ $(m=3,4, \ldots)$. The type and stability of bifurcation solutions depend on condition (C3) and the nonlinear property of system.

According to the classical Hopf bifurcation criterion mentioned above, the equivalent deterministic system (6) occurs as Hopf bifurcation if and only if (C1)-(C3) hold. To ensure that (C1) hold, the character polynomial (11) must exist as a pair of conjugate complex roots, and the parameters must satisfy one of the below conditions:

$$
\begin{array}{r}
a \bar{d}+a \delta<0 \\
a \bar{d}+2 a \delta<0 .
\end{array}
$$

To satisfy the eigenvalue's modules of (C1) conditions, we take

$$
\begin{gathered}
\left|\lambda_{1,2}\right|=| \pm \sqrt{a \bar{d}+a \delta}|=1, \\
\text { or }\left|\lambda_{3,4}\right|=| \pm \sqrt{a \bar{d}+2 a \delta}|=1 .
\end{gathered}
$$

By means of the MAPLE software, the relationships between the bifurcation parameter and the random strength are obtained:

$$
\begin{aligned}
\bar{d}_{1} & =-\frac{1}{a}-\delta \\
\text { or } \bar{d}_{2} & =-\frac{1}{a}-2 \delta .
\end{aligned}
$$

Substituting all expressions of (16) into character polynomial (11), respectively, all eigenvalues are as follows:

$$
\begin{aligned}
\lambda_{1} & =I, \\
\lambda_{2} & =-I, \\
\lambda_{3} & =\sqrt{-1+a \delta}, \\
\lambda_{4} & =-\sqrt{-1+a \delta}, \\
\text { or } \lambda_{1} & =I, \\
\lambda_{2} & =-I, \\
\lambda_{3} & =\sqrt{-1-a \delta}, \\
\lambda_{4} & =-\sqrt{-1-a \delta .}
\end{aligned}
$$

Obviously, because $\delta \geq 0$ and $2<a<3.5$, the eigenvalues that correspond to the parameter $\bar{d}_{2}=-1 / a-2 \delta$ do not satisfy the Hopf bifurcation condition (C1). However, when $0<a \delta<2$, the eigenvalues that correspond to the parameters $\bar{d}_{1}=\bar{d}_{c}=-1 / a-\delta$ can satisfy the Hopf bifurcation condition (C1). Therefore, there is only one expression $\bar{d}_{1}=\bar{d}_{c}=$ $-1 / a-\delta$, which can satisfy the Hopf bifurcation condition $(\mathrm{C} 1)$. In the meantime, $(\mathrm{C} 2)$ and $(\mathrm{C} 3)$ are written as

$$
\begin{aligned}
\frac{d\left|\lambda_{1}\left(\bar{d}_{c}\right)\right|}{d \bar{d}} & =\frac{1}{2 \sqrt{a \bar{d}_{c}+a \delta}} \neq 0, \\
\lambda_{1}^{3}\left(\bar{d}_{c}\right) & =\sqrt{a \bar{d}_{c}+a \delta} \neq 1, \\
\lambda_{1}^{m}\left(\bar{d}_{c}\right) & =\sqrt{a \bar{d}_{c}+a \delta} \neq 1 \\
& \left(m=4 k+1,4 k+2,4 k+3, k \in Z^{+}\right), \\
\text {or } \lambda_{1}^{m}\left(\bar{d}_{c}\right) & =\sqrt{a \bar{d}_{c}+a \delta}=1 \quad\left(m=4 k, k \in Z^{+}\right) .
\end{aligned}
$$




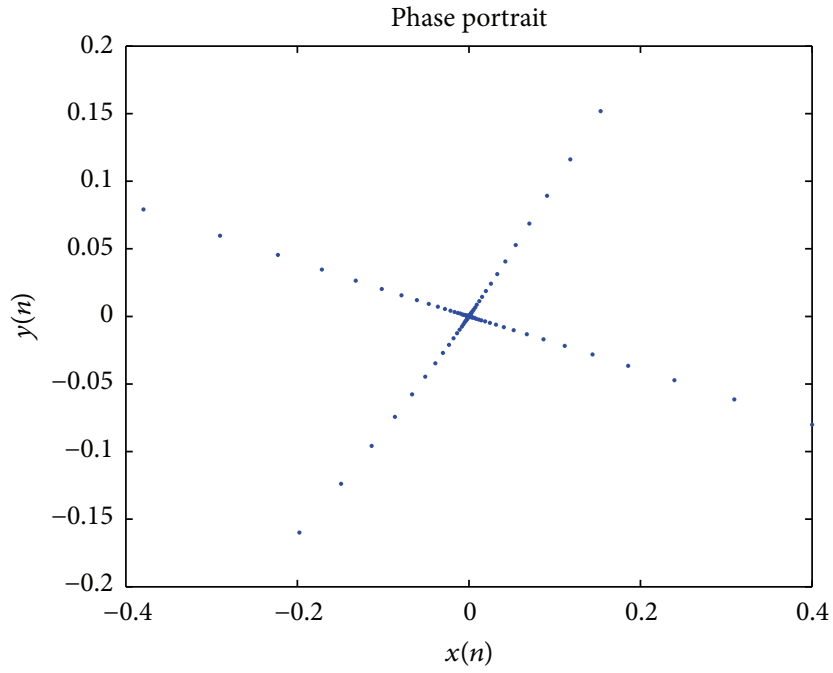

(a)

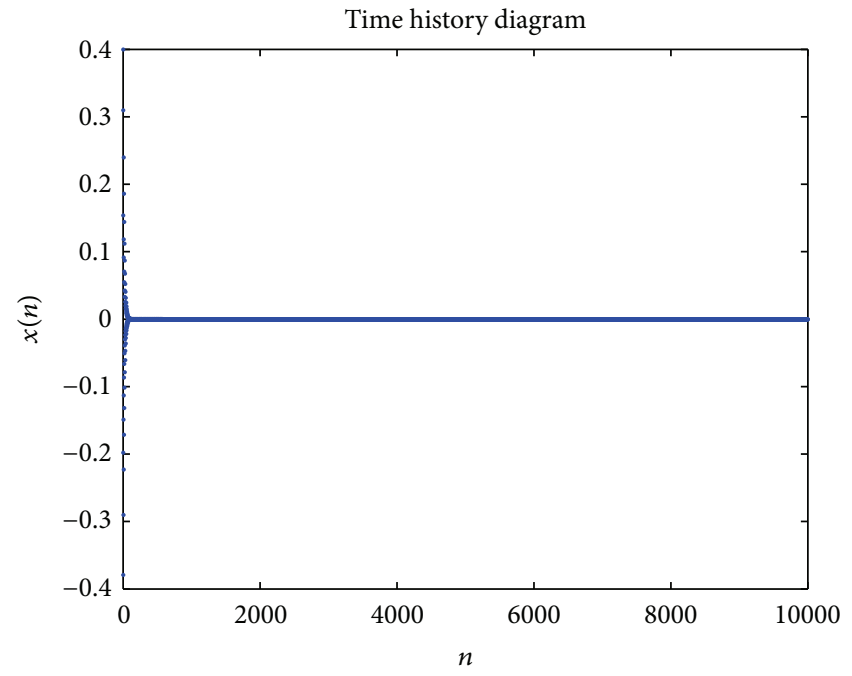

(b)

Figure 3: (a) Phase portrait and (b) time history diagram of equivalent deterministic system (6) with $\bar{d}=-0.45$.

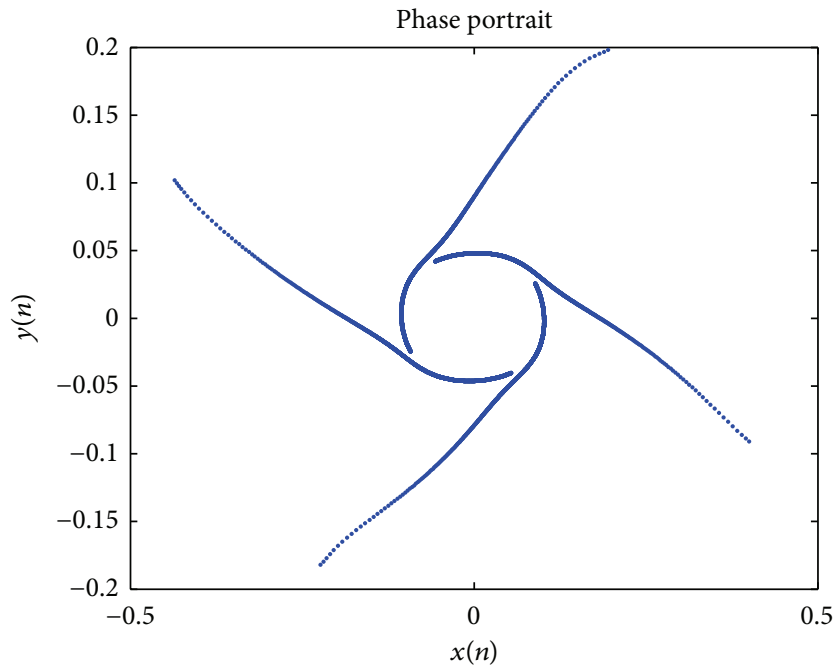

(a)

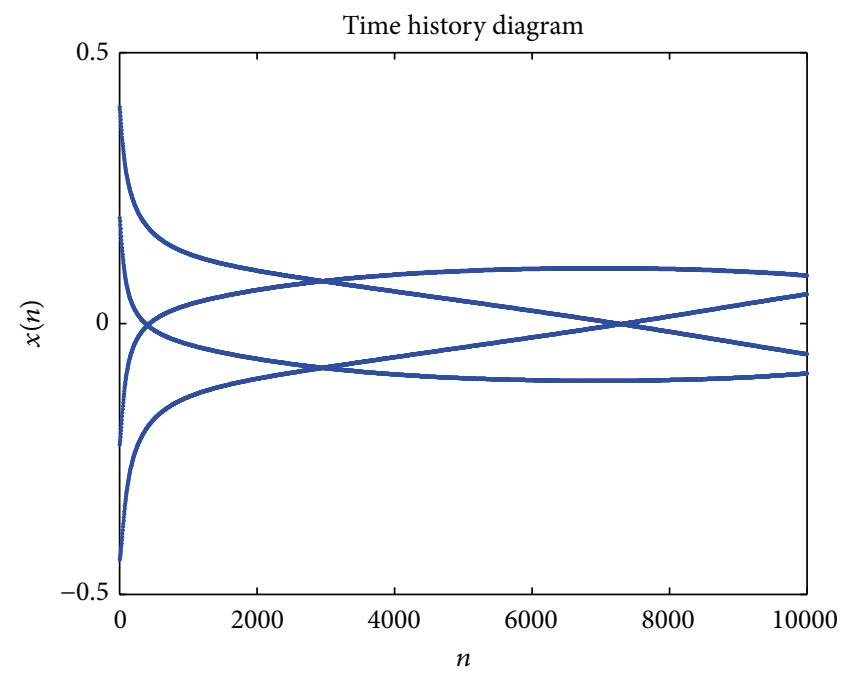

(b)

FIGURE 4: (a) Phase portrait and (b) time history diagram of equivalent deterministic system (6) with $\bar{d}=-0.5049$.

Thus, both (C2) and (C3) hold. According to the above analysis, when $0<a \delta<2$ and $\bar{d}=-1 / a-\delta$, all the conditions for the existence of the Hopf bifurcation hold. Because there is a pair of pure imaginary roots $\pm I$ in the eigenvalues, system (6) undergoes the Hopf bifurcations in the 1:4 strong resonance case. Numerical simulations are used to investigate equivalent deterministic system (6). Let $a=2.2, b=-0.1$, and $c=-0.9$, and random strength is given by $\delta=0.05$, and the parameter $\bar{d}$ is given by $-0.45,-0.5049$, and -0.508 , respectively. Phase portraits and time history diagrams are displayed in Figures 3, 4, and 5, respectively.

\section{The Amplitude Control for Hopf Bifurcation}

A method is introduced to control the amplitude of Hopf bifurcation, which does not require any adjustable control 


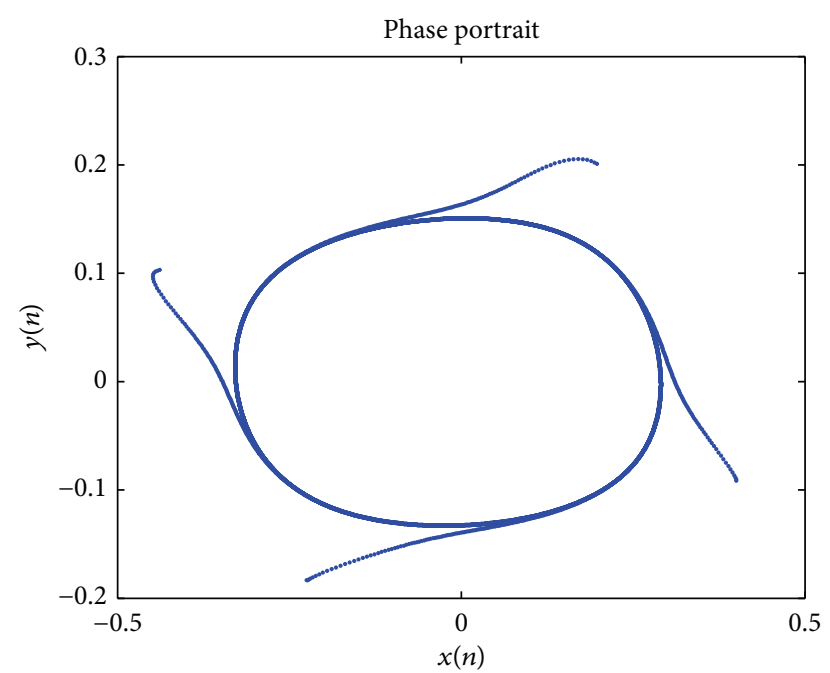

(a)

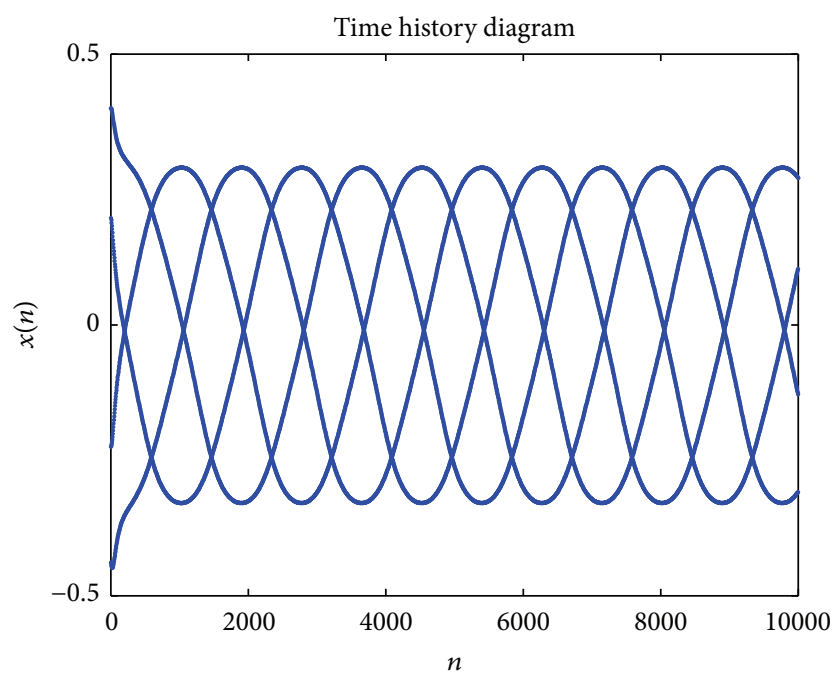

(b)

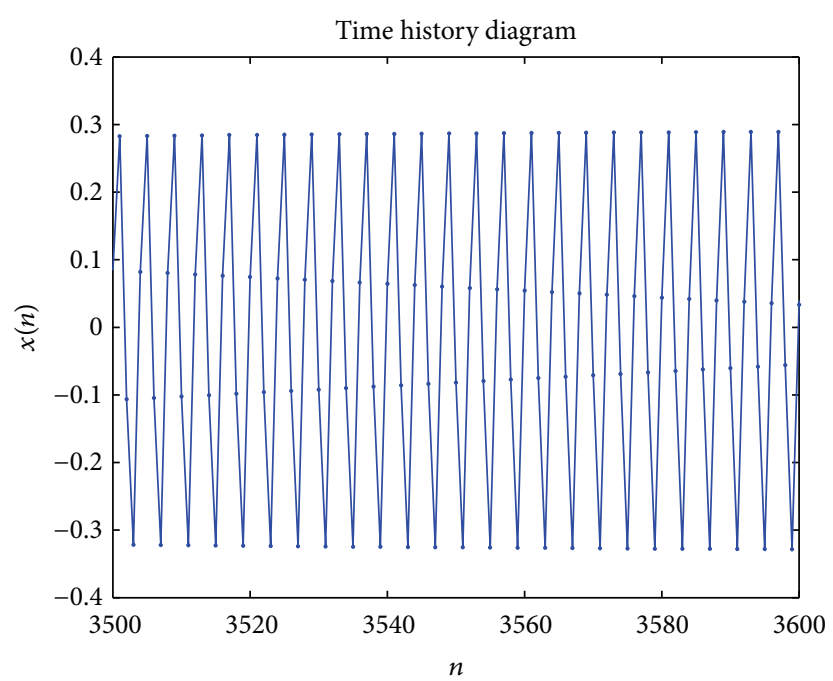

(c)

Figure 5: (a) Phase portrait and (b) time history diagram of equivalent deterministic system (6) with $\bar{d}=-0.508$. (c) Local amplification corresponding to (b).

parameters of the system. Our objective here is to research the influence of random intensity for the Hopf bifurcation amplitude of equivalent deterministic system (6). When parameter $\bar{d}=-0.508$ and random intensity $\delta=0.05$, respectively, equivalent deterministic system (6) occurs as the Hopf bifurcation. As the random intensity increases to $\delta=0.051$, the amplitude of limit cycle in equivalent deterministic system (6) is becoming smaller; the phase trajectories and time history diagrams are shown as in Figure 6. Increasing the random intensity to $\delta=0.052$, the amplitude of limit cycle in equivalent deterministic system (6) is more smaller than the amplitude of limit cycle of Figure 6; the phase trajectories and time history diagrams are shown in Figure 7 . As we continue to increase random intensity to $\delta=0.0528$, the amplitude of limit cycle in equivalent deterministic system (6) is more smaller than the amplitude of limit cycle of Figure 7; the phase trajectories and time history diagrams are shown in Figure 8.

According to the numerical simulations, we can find that amplitude of the stochastic discrete-time hyperchaotic system will be controlled by changing the random intensity. Compared to the deterministic system, the random intensity 


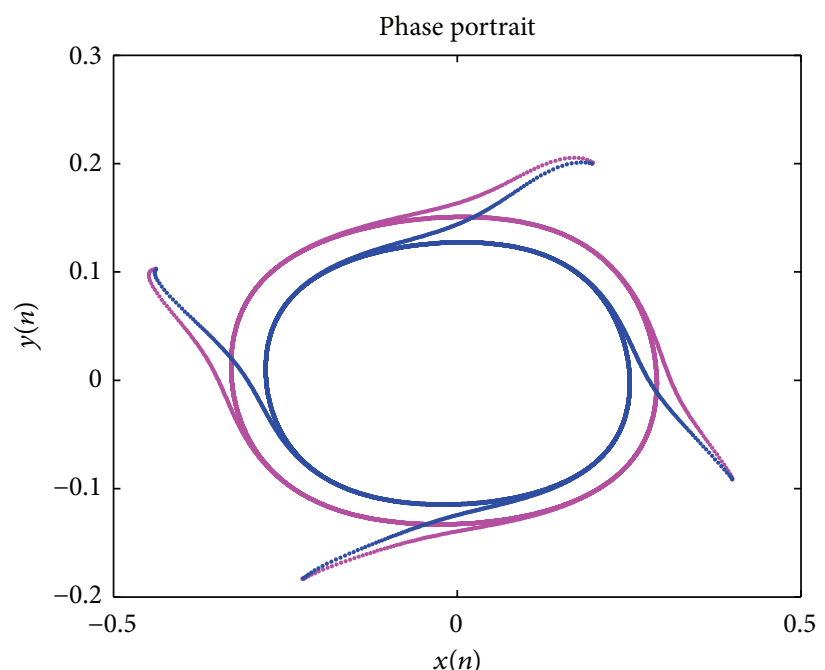

. $\delta=0.05$

. $\delta=0.051$

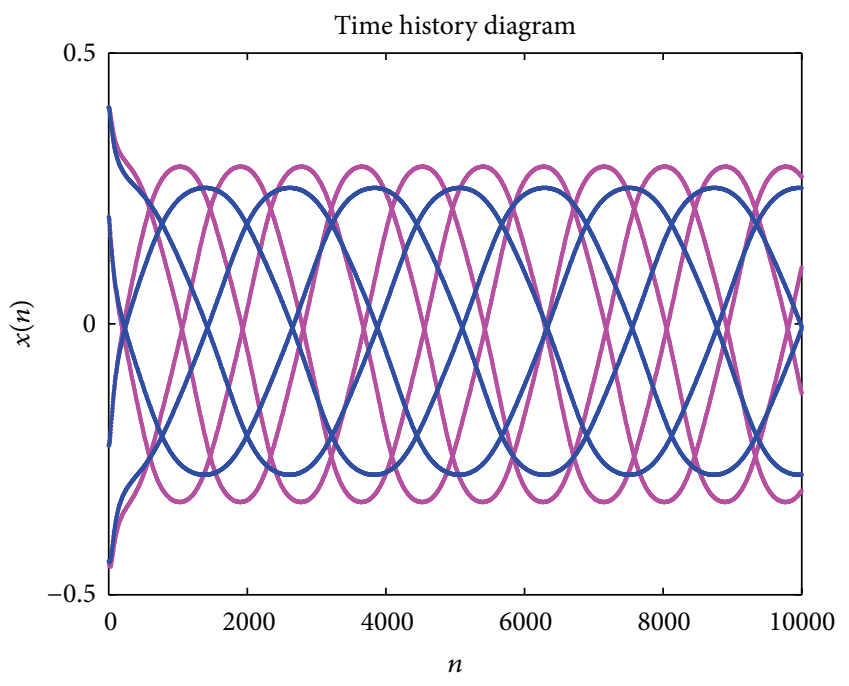

. $\delta=0.05$

- $\delta=0.051$

(a)

(b)

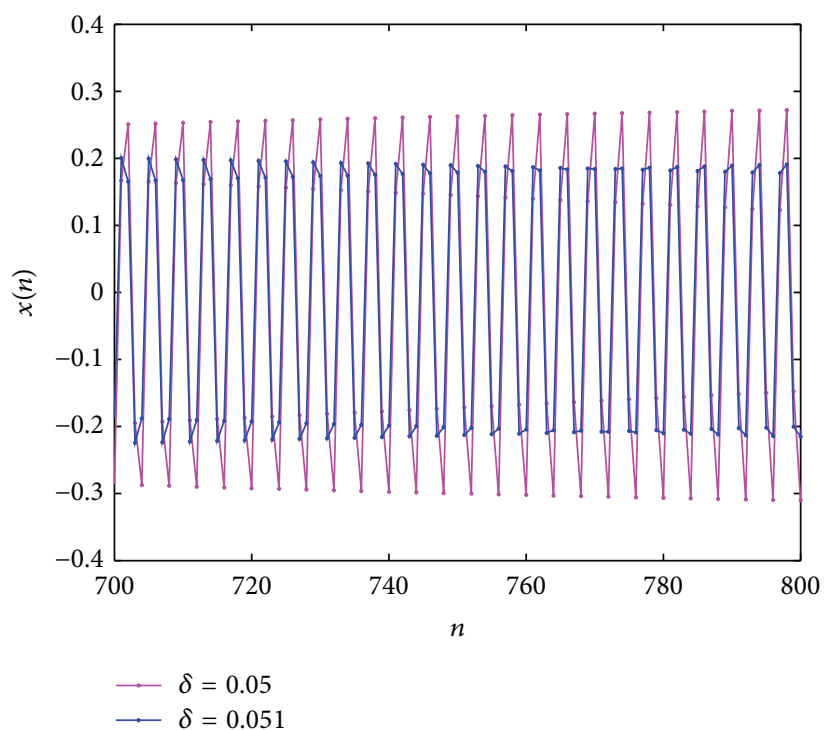

(c)

Figure 6: (a) Phase portrait and (b) time history diagram of stochastic discrete-time hyperchaotic system (6) with $\delta=0.05$ and $\delta=0.051$. (c) Local amplification corresponding to (b).

obviously affects the bifurcation amplitude of its stochastic system, and the bifurcation amplitude decreases with the random intensity. Numerical simulations are used to investigate equivalent deterministic system (6). Here, we take $a=2.2$, $b=-0.1$, and $c=-0.9$, and the equivalent deterministic system undergoes strong resonance Hopf bifurcation at the fixed point, when the bifurcation parameter $\bar{d}=$ -0.508 . The phase trajectories and time history diagrams for the stochastic discrete-time hyperchaotic system are depicted in Figures 6, 7, and 8.

\section{Conclusions}

In this paper, numerical simulations are presented to illustrate our results with the theoretical analysis and to exhibit the complex dynamical behaviors. Furthermore, the amplitude 


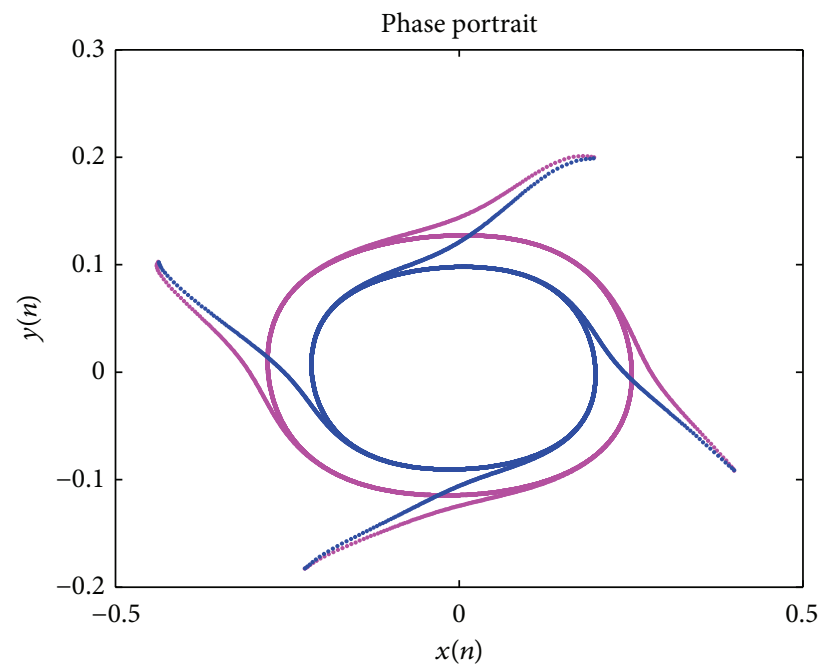

- $\delta=0.051$

- $\delta=0.052$

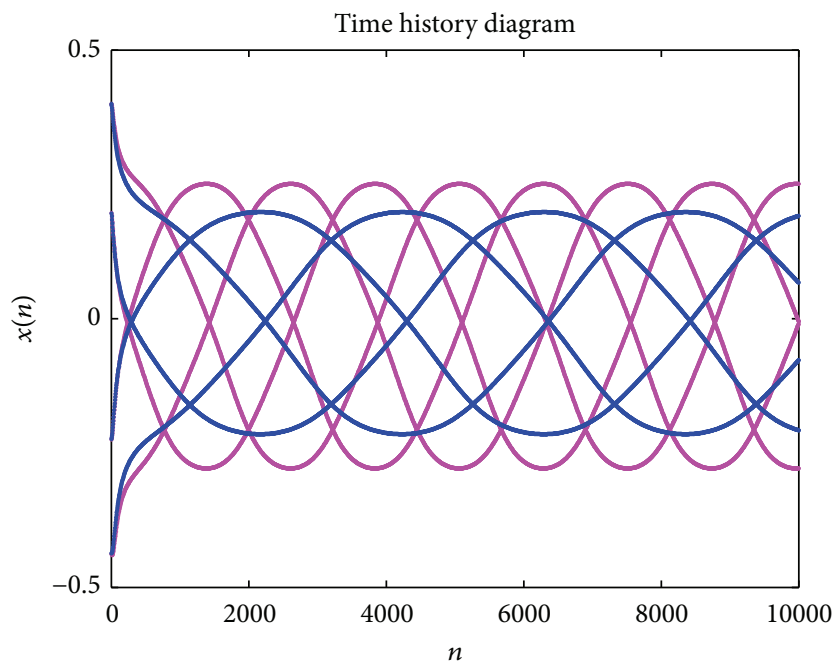

- $\delta=0.051$

. $\delta=0.052$

(a)

(b)

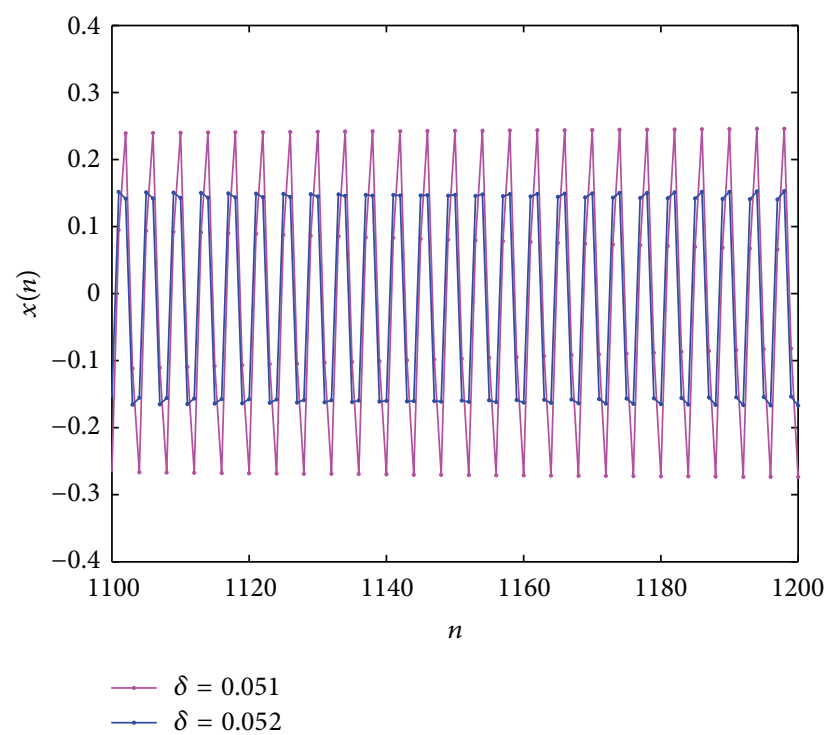

(c)

Figure 7: (a) Phase portrait and (b) time history diagram of stochastic discrete-time hyperchaotic system (6) with $\delta=0.051$ and $\delta=0.052$. (c) Local amplification corresponding to (b).

control of Hopf bifurcation of a stochastic discrete-time hyperchaotic system has been analyzed. Stochastic discretetime hyperchaotic system undergoes strong resonance Hopf bifurcation at a fixed point by means of the Hopf bifurcation theory. In addition, a random intensity control law has been introduced for the stochastic discrete-time hyperchaotic system. The amplitude of the Hopf bifurcation can be controlled by changing the random intensity. We can find that the effect of the random intensity on controlling the Hopf bifurcation is available. Numerical simulations show the effectiveness of the analytical results.

\section{Appendix}

$S_{i}(n)(i=0,1)$ and $U_{i}(n)(i=0,1)$ in system (6) can be derived through the MAPLE as follows:

$$
\begin{aligned}
& S_{0}(n)=b\left(x_{0}^{2}(n)+x_{1}^{2}(n)\right), \\
& S_{1}(n)=b\left(2 x_{0}(n) x_{1}(n)+x_{1}^{2}(n)\right), \\
& U_{0}(n)=c\left(y_{0}^{2}(n)+y_{1}^{2}(n)\right),
\end{aligned}
$$



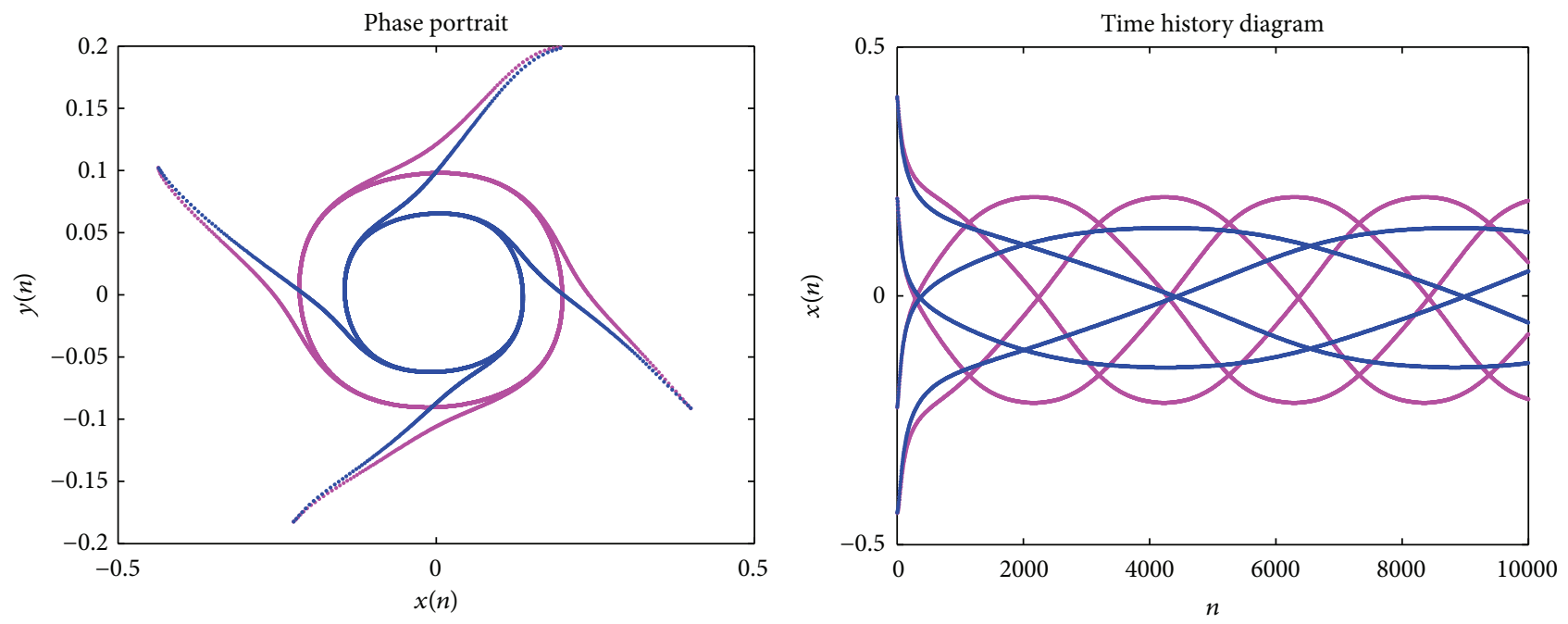

$\delta=0.052$
$\cdot \delta=0.0528$

- $\delta=0.052$

$\cdot \delta=0.0528$

(a)

(b)

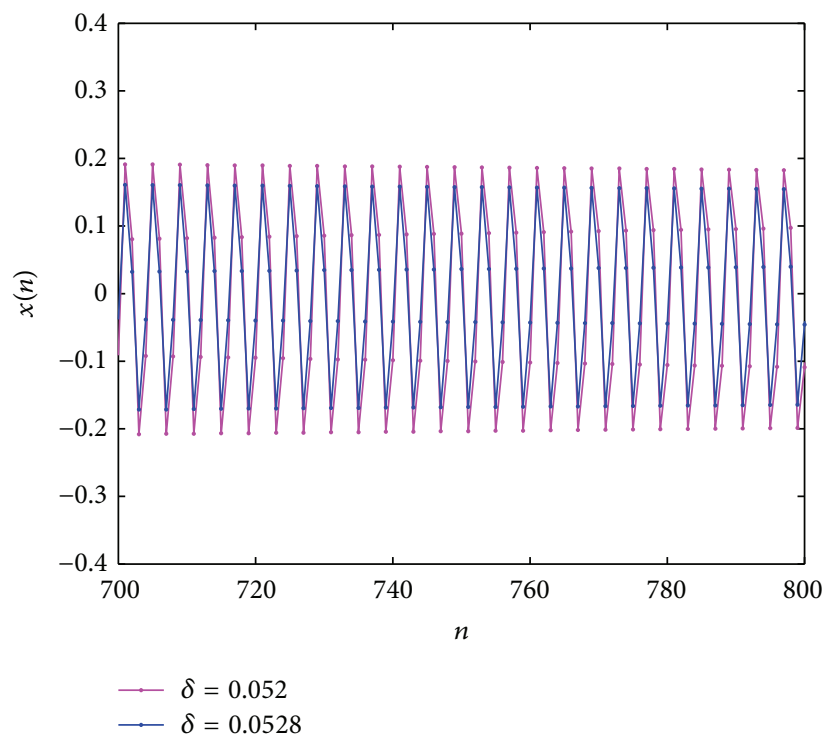

(c)

Figure 8: (a) Phase portrait and (b) time history diagram of stochastic discrete-time hyperchaotic system (6) with $\delta=0.052$ and $\delta=0.0528$. (c) Local amplification corresponding to (b).

$$
U_{1}(n)=c\left(2 y_{0}(n) y_{1}(n)+y_{1}^{2}(n)\right) \text {. }
$$

\section{Conflict of Interests}

All authors declare that there is no conflict of interests regarding the publication of this paper.

\section{References}

[1] H. T. Zhao, Y. P. Lin, and Y. X. Dai, "Bifurcation analysis and control of chaos for a hybrid ratio-dependent three species food chain," Applied Mathematics and Computation, vol. 218, no. 5, pp. 1533-1546, 2011.

[2] N. Yi, P. Liu, and Q. L. Zhang, "Bifurcations analysis and tracking control of an epidemic model with nonlinear incidence 
rate," Applied Mathematical Modelling: Simulation and Computation for Engineering and Environmental Systems, vol. 36, no. 4, pp. 1678-1693, 2012.

[3] J. Guckenheimer and P. Holmes, Nonlinear Oscillations, Dynamical Systems and Bifurcations of Vector Fields, Springer, New York, NY, USA, 1986.

[4] E. I. Jury, Theory and Application of the Z-Transform Method, Wiley, New York, NY, USA, 1964.

[5] Y. A. Kuznetsov, Elements of Applied Bifurcation Theory, Springer, New York, NY, USA, 2nd edition, 1998.

[6] B. D. Hassard, N. D. Kazarinoff, and Y. Wan, Theory and Application of Hopf Bifurcation, Cambridge University Press, Cambridge, UK, 1981.

[7] G. Chen, J. Lu, B. Nicholas, and S. M. Ranganathan, "Bifurcation dynamics in discrete-time delayed-feedback control systems," International Journal of Bifurcation and Chaos, vol. 9, no. 1, pp. 287-293, 1999.

[8] E. H. Abed and H. O. Wang, "Feedback control of bifurcation and chaos in dynamical Systems," in Nonlinear Dynamics and Stochastic Mechanics, pp. 153-173, CRC Press, Boca Raton, Fla, USA, 1995.

[9] Z. Q. Wu and L. M. Sun, "Hopf bifurcation control of the system based on washout filter controller," Acta Physica Sinica, vol. 60, no. 5, pp. 1-5, 2011.

[10] G. Wen, "Criterion to identify Hopf bifurcations in maps of arbitrary dimension," Physical Review E, vol. 72, no. 2, Article ID 026201, 4 pages, 2005.

[11] G. L. Wen, D. L. Xu, and J. H. Xie, "Control of degenerate Hopf bifurcations in three-dimensional maps," Chaos, vol. 13, no. 2, pp. 486-494, 2003.

[12] O. E. Rössler, "An equation for hyperchaos," Physics Letters A, vol. 71, no. 2-3, pp. 155-157, 1979.

[13] H. X. Wang, G. L. Cai, S. Miao, and L. X. Tian, "Nonlinear feedback control of a novel hyperchaotic system and its circuit implementation," Chinese Physics B, vol. 19, no. 3, Article ID 030509, pp. 1-8, 2010.

[14] Y. Li, W. K. S. Tang, and G. Chen, "Generating hyperchaos via state feedback control," International Journal of Bifurcation and Chaos in Applied Sciences and Engineering, vol. 15, no. 10, pp. 3367-3375, 2005.

[15] X. P. Guan, Z. P. Fan, and C. L. Chen, Chaos Control and Its Application in Secure Communication, National Defence Industry Press, Beijing, China, 2002.

[16] K. Sun, X. Liu, C. Zhu, and J. C. Sprott, "Hyperchaos and hyperchaos control of the sinusoidally forced simplified Lorenz system," Nonlinear Dynamics, vol. 69, no. 3, pp. 1383-1391, 2012.

[17] H. Yu, G. Cai, and Y. Li, "Dynamic analysis and control of a new hyperchaotic finance system," Nonlinear Dynamics, vol. 67, no. 3, pp. 2171-2182, 2012.

[18] C. Li, X. Liao, and K.-W. Wong, "Lag synchronization of hyperchaos with application to secure communications," Chaos, Solitons \& Fractals, vol. 23, no. 1, pp. 183-193, 2005.

[19] J. H. Ma and Y. J. Yang, "Hyperchaos numerical simulation and control in a 4D hyperchaotic system," Discrete Dynamics in Nature and Society, vol. 2013, Article ID 980578, 16 pages, 2013.

[20] K. B. Deng and S. M. Yu, "Hopf bifurcation analysis of a new modified hyperchaotic Lü system," Optik, vol. 124, no. 23, pp. 6265-6269, 2013.
[21] X. L. Leng, C. L. Wu, X. P. Ma, G. Meng, and T. Fang, "Bifurcation and chaos analysis of stochastic Duffing system under harmonic excitations," Nonlinear Dynamics, vol. 42, no. 2, pp. 185-198, 2005.

[22] S.-J. Ma, W. Xu, W. Li, and T. Fang, "Analysis of stochastic bifurcation and chaos in stochastic Duffing-van der Pol system via Chebyshev polynomial approximation," Chinese Physics, vol. 15, no. 6, pp. 1231-1238, 2006.

[23] S. J. Ma, W. Xu, and T. Fang, "Analysis of period-doubling bifurcation in double-well stochastic Duffing system via Laguerre polynomial approximation," Nonlinear Dynamics, vol. 52, no. 3, pp. 289-299, 2008.

[24] S. J. Ma and W. Xu, "Period-doubling bifurcation in an extended van der Pol system with bounded random parameter," Communications in Nonlinear Science and Numerical Simulation, vol. 13, no. 10, pp. 2256-2265, 2008.

[25] Y. Xu, S. Ma, and H. Zhang, "Hopf bifurcation control for stochastic dynamical system with nonlinear random feedback method," Nonlinear Dynamics, vol. 65, no. 1-2, pp. 77-84, 2011. 


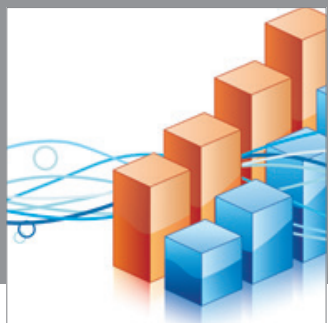

Advances in

Operations Research

mansans

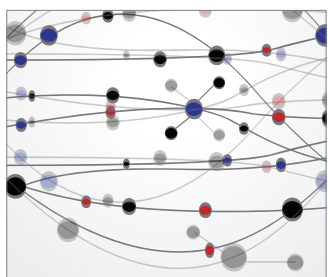

The Scientific World Journal
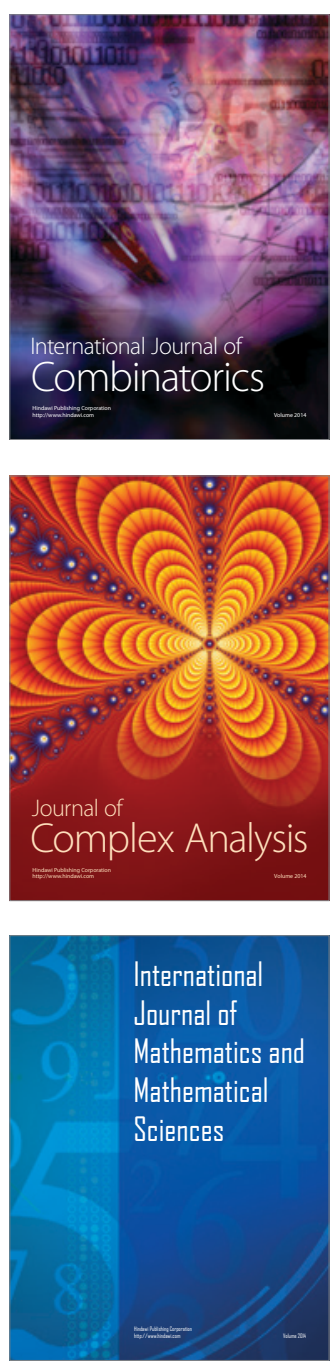
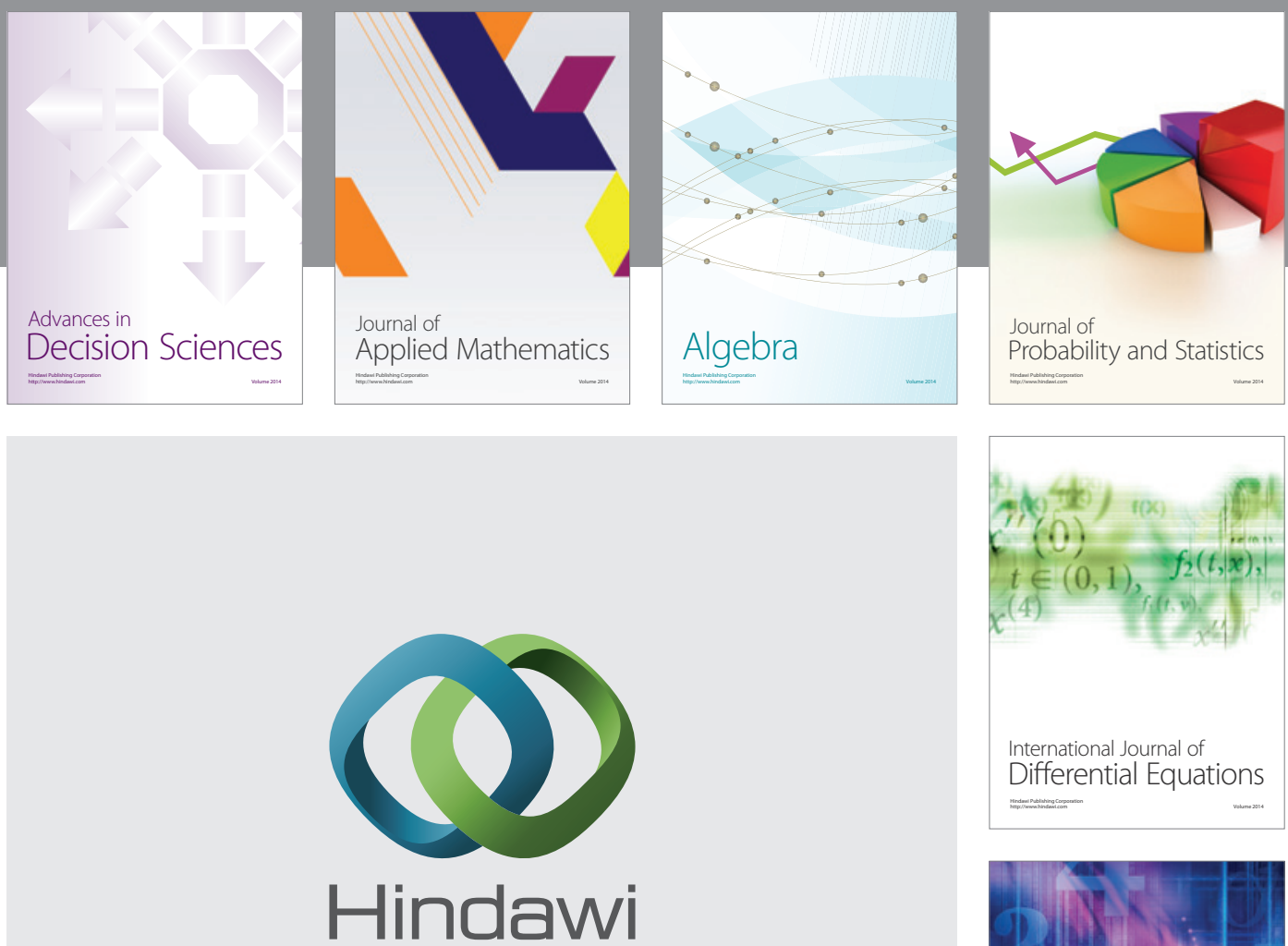

Submit your manuscripts at http://www.hindawi.com
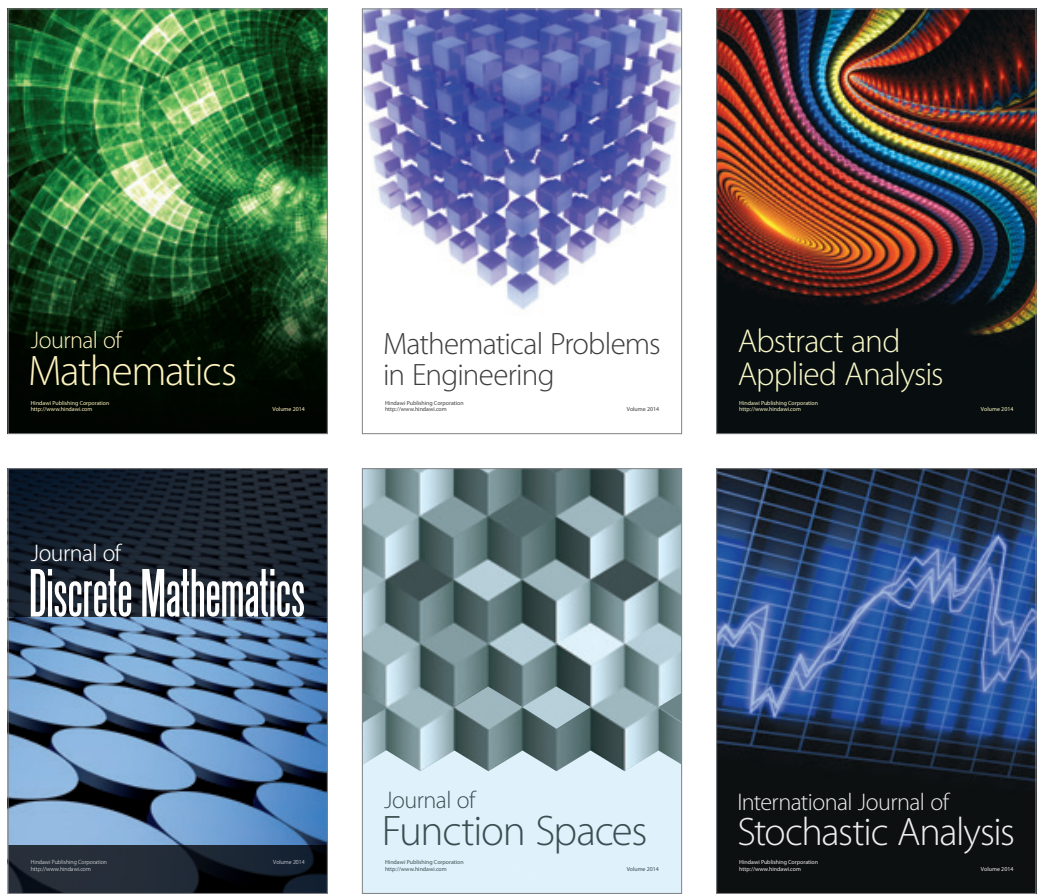

Journal of

Function Spaces

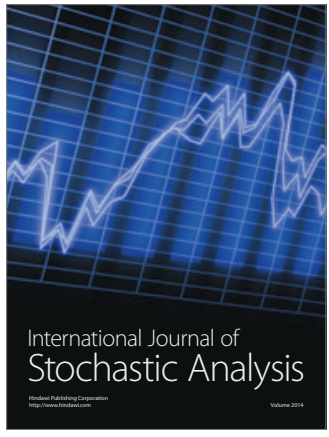

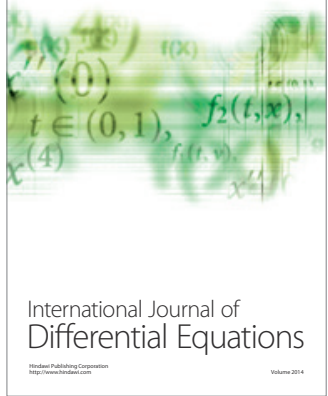
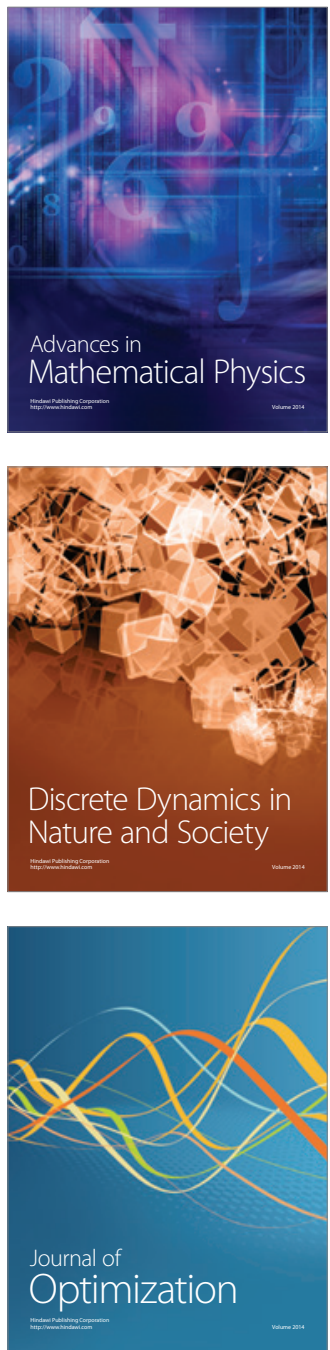